\title{
Comprehension-Based Versus Production-Internal Feedback in Planning Spoken Words: A Rejoinder to Rapp and Goldrick (2004)
}

\author{
Ardi Roelofs \\ Max Planck Institute for Psycholinguistics and F. C. Donders Centre for Cognitive Neuroimaging
}

\begin{abstract}
WEAVER + + has no backward links in its form-production network and yet is able to explain the lexical and mixed error biases and the mixed distractor latency effect. This refutes the claim of B. Rapp and M. Goldrick (2000) that these findings specifically support production-internal feedback. Whether their restricted interaction account model can also provide a unified account of the error biases and latency effect remains to be shown.
\end{abstract}

There were two major points in my comment (Roelofs, 2004) on the article by Rapp and Goldrick (2000). My first major point was that the lexical and mixed error biases and the mixed distractor latency effect do not specifically support production-internal feedback, contrary to what Rapp and Goldrick (2000) maintained. I showed that a model with comprehension-based rather than production-internal feedback, namely WEAVER + + (e.g., Levelt, Roelofs, \& Meyer, 1999; Roelofs, 1992, 1997, 2003a, 2003b; Roelofs \& Hagoort, 2002), accounts for the error and latency findings. My second major point was that extant productioninternal feedback accounts of the error biases and latency effects are incompatible.

In their reply, Rapp and Goldrick (2004) agreed that the empirical evidence leaves open whether the feedback is comprehension based or production internal: "We find no clear evidence in support of the claim that feedback in spoken word production is unambiguously a component of the comprehension process. However, it is important to be clear that neither is there clear evidence that it is not" (p. 578). Moreover, they were silent on the issue of whether the production-internal feedback in their restricted interaction account (RIA) model serves speech comprehension. It is important to be clear, however, that regardless of the possible comprehension purposes of the backward links in RIA, the account of the findings by Rapp and Goldrick (2000) is in terms of feedback that occurs in a production network, which differs from my account in terms of two strictly feedforward networks, one for production and the other for comprehension, as implemented in WEAVER + +. I refer to Roelofs (2003b) for a review of the evidence for distinct word-form networks for production and comprehension. On my account, feedback arises because the feedforward production network activates the feedforward comprehension network. A secondary issue is whether activation of the comprehension network by the production network happens automatically, as suggested by the new evidence of Humphreys (2002) and

Ardi Roelofs, Max Planck Institute for Psycholinguistics, Nijmegen, the Netherlands, and F. C. Donders Centre for Cognitive Neuroimaging, Nijmegen, the Netherlands.

Correspondence concerning this article should be addressed to Ardi Roelofs, Max Planck Institute for Psycholinguistics, Wundtlaan 1, 6525 XD, Nijmegen, the Netherlands. E-mail: ardi@mpi.nl
Nooteboom (in press) referred to by Rapp and Goldrick (2004), or whether it is under voluntary control, as I assumed on the basis of the available evidence (Roelofs, 2004). Either way, the feedback does not happen production-network internally, which is the critical point.

The second major point of my comment (Roelofs, 2004) was that extant production-internal feedback accounts of the mixed error bias and the mixed distractor latency effect are incompatible. Rapp and Goldrick (2004) disputed this claim. According to them, in the context of the mixed error effect, the activation of a mixed neighbor (e.g., calf in naming a cat) will be higher than that of a semantic neighbor (e.g., dog). This is because production-internal feedback from the form of cat activates the lemma of calf but not the lemma of $d o g$, which makes erroneous selection of calf for cat more likely than erroneous selection of $d o g$ for cat. The situation in a picture-word interference experiment was argued to be different. Here, the issue was said to be which distractor $(C A L F$ or $D O G$ ) is most likely to increase the activation of the target (the lemma of cat) and therefore to reduce the time needed for its selection. Production-internal feedback from the form of the distractor $C A L F$ activates the lemma of cat, whereas feedback from the distractor $D O G$ does not, which reduces the semantic interference of $C A L F$ compared with $D O G$ in planning to say "cat."

The problem with this account, however, is that the lemma of cat also activates the form and, through feedback, the lemma of calf (assumed to explain the mixed error effect) when $C A L F$ is presented as distractor. Consequently, the level of activation of the lemma of calf is enhanced compared with the activation of the lemma of $d o g$ in planning to say "cat," both in the context of the mixed error effect and in the picture-word interference situation. Given this, it remains unclear why mixed items yield more competition and hence a larger number of errors than items that are semantically related only, whereas mixed distractors yield less competition and hence shorter latencies than distractors that are semantically related only. Thus, the challenge remains to show that production-internal feedback explains both the error bias and the latency effect.

In conclusion, WEAVER ++ has no backward links in its production network and yet is able to account for the lexical and mixed error biases and the mixed distractor latency effect. This refutes the claim of Rapp and Goldrick (2000) that the error biases and latency effects specifically support production-internal feed- 
back. Whether the RIA model of Rapp and Goldrick (2000) can provide a unified account of the error biases and latency effects remains to be shown.

\section{References}

Levelt, W. J. M., Roelofs, A., \& Meyer, A. S. (1999). A theory of lexical access in speech production. Behavioral and Brain Sciences, 22, 1-38.

Rapp, B., \& Goldrick, M. (2000). Discreteness and interactivity in spoken word production. Psychological Review, 107, 460-499.

Rapp, B., \& Goldrick, M. (2004). Feedback by any other name is still interactivity: A reply to Roelofs (2004). Psychological Review, 111, 573-578.

Roelofs, A. (1992). A spreading-activation theory of lemma retrieval in speaking. Cognition, 42, 107-142.

Roelofs, A. (1997). The WEAVER model of word-form encoding in speech production. Cognition, 64, 249-284.
Roelofs, A. (2003a). Goal-referenced selection of verbal action: Modeling attentional control in the Stroop task. Psychological Review, 110, 88 125 .

Roelofs, A. (2003b). Modeling the relation between the production and recognition of spoken word forms. In A. S. Meyer \& N. O. Schiller (Eds.), Phonetics and phonology in language comprehension and production: Differences and similarities (pp. 115-158). Berlin, Germany: Mouton de Gruyter.

Roelofs, A. (2004). Error biases in spoken word planning and monitoring by aphasic and nonaphasic speakers: Comment on Rapp and Goldrick (2000). Psychological Review, 111, 561-572.

Roelofs, A., \& Hagoort, P. (2002). Control of language use: Cognitive modeling of the hemodynamics of Stroop task performance. Cognitive Brain Research, 15, 85-97.

Received July 22, 2003

Accepted August 7, 2003

\section{Members of Underrepresented Groups: Reviewers for Journal Manuscripts Wanted}

If you are interested in reviewing manuscripts for APA journals, the APA Publications and Communications Board would like to invite your participation. Manuscript reviewers are vital to the publications process. As a reviewer, you would gain valuable experience in publishing. The $\mathrm{P} \& \mathrm{C}$ Board is particularly interested in encouraging members of underrepresented groups to participate more in this process.

If you are interested in reviewing manuscripts, please write to Demarie Jackson at the address below. Please note the following important points:

- To be selected as a reviewer, you must have published articles in peer-reviewed journals. The experience of publishing provides a reviewer with the basis for preparing a thorough, objective review.

- To be selected, it is critical to be a regular reader of the five to six empirical journals that are most central to the area or journal for which you would like to review. Current knowledge of recently published research provides a reviewer with the knowledge base to evaluate a new submission within the context of existing research.

- To select the appropriate reviewers for each manuscript, the editor needs detailed information. Please include with your letter your vita. In your letter, please identify which APA journal(s) you are interested in, and describe your area of expertise. Be as specific as possible. For example, "social psychology" is not sufficient-you would need to specify "social cognition" or "attitude change" as well.

- Reviewing a manuscript takes time (1-4 hours per manuscript reviewed). If you are selected to review a manuscript, be prepared to invest the necessary time to evaluate the manuscript thoroughly.

Write to Demarie Jackson, Journals Office, American Psychological Association, 750 First Street, NE, Washington, DC 20002-4242. 